\title{
L'apport de la loi de finances 2018 au droit social algérien
}

\section{Chakib Boukli Hacène}

\section{Q OpenEdition}

1 Journals

\section{Édition électronique}

URL : https://journals.openedition.org/rdctss/2039

DOI : $10.4000 /$ rdctss.2039

ISSN : 2262-9815

Éditeur

Centre de droit comparé du travail et de la sécurité sociale

\section{Édition imprimée}

Date de publication : 1 avril 2018

Pagination : 116-119

ISSN : 2117-4350

\section{Référence électronique}

Chakib Boukli Hacène, "L'apport de la loi de finances 2018 au droit social algérien », Revue de droit comparé du travail et de la sécurité sociale [En ligne], 1 | 2018, mis en ligne le 01 novembre 2021, consulté le 12 novembre 2021. URL : http://journals.openedition.org/rdctss/2039 ; DOI : https:// doi.org/10.4000/rdctss.2039

\section{(c) (i) $\odot$}

Revue de droit comparé du travail et de la sécurité sociale est mise à disposition selon les termes de la Licence Creative Commons Attribution - Pas d'Utilisation Commerciale - Pas de Modification 4.0 International. 


\section{CHAKIB BOUKLI-HACENE}

UNIVERSITÉ DE SAÏDA

\section{L'APPORT DE LA LOI DE FINANCES 2018 AU DROIT SOCIAL ALGÉRIEN}

L'actualité juridique en droit social algérien de ce premier semestre 2018, réside dans la nouvelle loi de finances promulguée à la fin du mois de décembre 20171. Il est vrai qu'une loi de finances consiste en temps normal, à prévoir et autoriser pour chaque année civile, l'ensemble des ressources et des charges de l'Etat, ainsi que les autres moyens financiers destinés au fonctionnement des services publics ${ }^{2}$. Elle devrait donc se limiter à ses missions fixées par la loi.

Cependant, le législateur algérien semble avoir pris, depuis un certain temps, I'habitude de règlementer au sein même des lois de finances des sujets totalement étrangers à la budgétisation de l'Etat. A cet égard, les lois de finances, en Algérie, sont devenues un "recueil de lois de fin d'année» comportant en plus des textes sur la gestion des fonds publics, d'autres textes sans relation avec ces derniers, que les gouvernements successifs, sans raison apparente et de manière inattendue, n'ont pas souhaité proposer dans des textes de lois distincts. Cette pratique curieuse ne parait pas vraiment justifiée.

La loi de finances de cette année ne fait pas exception. Elle contient en effet, des textes de différentes disciplines juridiques sans aucune relation avec le budget de l'Etat, comme le droit immobilier, le droit commercial, le droit bancaire et aussi le droit social. En effet, cette nouvelle loi de finances modifie et complète des textes de loi relatifs à la fois au droit de la sécurité sociale (I) et au droit du travail (II).

\section{I - L'APPORT DE LA LOI DE FINANCES 2018 AU DROIT DE LA SÉCURITÉ SOCIALE}

En droit algérien, conformément à la loi $83-14^{3}$, il est fait obligation aux bénéficiaires des prestations de sécurité sociale d'adresser à l'organisme compétent une déclaration d'activité dans les dix (10) jours qui suivent le début d'exercice de la dite activité. Outre les employeurs et autres bénéficiaires de la sécurité sociale, sont également soumis à l'obligation de déclaration d'activité, suivant l'article 5 de la même loi, les personnes exerçant pour leur propre compte une activité professionnelle, industrielle, commerciale, agricole, artisanale, libérale, même si elles n'occupent pas de personnel salarié. Pour ces derniers, la déclaration d'activité vaut demande d'affiliation aux différents organismes de la sécurité sociale.

2 Voir art. 3 de la loi n 84-17 du 07/07/1984, relative à la loi de finances, modifié et complété par la loi n 88-05 du 12/01/1988.

3 Voir loi n 83-14 du 02/07/1983, relative aux obligations des assujettis en matière de sécurité sociale, JORA n² $28 / 1983$. 
Cependant, l'article 97 de la loi de finances 2018 apporte un léger aménagement à la loi de 1983 précitée, concernant les personnes exerçant une activité commerciale. II dispose que : "l'immatriculation au registre du commerce des personnes exerçant une activité commerciale pour leur propre compte, vaut déclaration au régime de sécurité sociale des non-salarié ... ». En effet, uniquement pour cette catégorie professionnelle, l'affiliation à l'organisme de la sécurité sociale des non-salariés, ne résulte plus d'une déclaration d'activité dans les 10 jours qui suivent le début de l'exercice, comme prévu pour les autres personnes suscitées, elle devient automatique du fait simplement de l'immatriculation au registre du commerce.

Il semblerait que cet amendement ait été introduit pour alléger les procédures d'affiliation des commerçants à la sécurité sociale, et aussi pour réduire le champ des activités informelles ${ }^{4}$. II aurait cependant été plus judicieux d'étendre cette nouvelle procédure à toutes les autres catégories professionnelles. Reste à savoir, si ce modeste aménagement ne constitue qu'un cas isolé, ou s'agirait-il de la première étape d'un grand plan de modernisation des services d'affiliation à la sécurité sociale?

\section{II - L'APPORT DE LA LOI DE FINANCES AU DROIT DU TRAVAIL}

C'est un apport à caractère purement pénal, qui consiste en une révision à la hausse des différentes peines prévues pour toute violation de certaines règles d'hygiène, de sécurité et de médecine du travail ainsi que pour toute infraction aux dispositions de la loi sur les relations du travail.

En effet, suite à la dépréciation de la monnaie nationale (le Dinar algérien) de manière continue ces trois dernières décennies ${ }^{5}$, les amendes délictuelles et contraventionnelles prévues par le Code pénal sont devenues désuètes et dépourvues de tout pouvoir coercitif. C'est pourquoi, le législateur algérien a modifié en $2006^{6}$ l'article 5 du Code pénal, en augmentant le montant des peines d'amende des délits et des contraventions. L'amende délictuelle a été fixée à plus de vingt mille (20.000) dinars contre deux mille (2.000) dinars auparavant ; quant à l'amende contraventionnelle, elle a été déterminée dans une fourchette qui oscille entre deux mille (2.000) et vingt mille (20.000) dinars, contre vingt (20) et deux mille (2.000) dinars précédemment.

Cet aménagement devait engendrer obligatoirement la révision de tous les textes de loi qui comportent des peines d'amende délictuelle ou contraventionnelle, afin de les aligner avec les nouvelles dispositions du Code pénal. Mais, au lieu de procéder à une vaste opération de mise à niveau de tous ces textes de loi, en prenant en compte chaque législation, le législateur algérien a choisi d'effectuer des aménagements avec parcimonie.

4 Voir sur ce sujet, M. N.-E. Koriche, « La part du droit dans la formalisation du droit en Algérie : une diversité de mesures sans stratégie claire », Revue de droit comparé du travail et de sécurité sociale, $n^{\circ}$ 3/2017, pp. 87-88.

5 En 1990, 1 dollars américain valait 12,02 dinars, alors qu'en 2018, il vaut 113,756 dinars (sources Banque d'Algérie).

6 Voir loi n 06-23 du 20/12/2006, modifiant et complétant l'ordonnance n 66-156 du 08/06/1966 portant Code pénal, JORA n 84/2006. 
C'est ce qui explique que la plus grande partie des peines d'amende prévues dans la législation du travail algérien, sont restées sans changement depuis leur promulgation en 1990 ; elles ont de ce fait perdu leur effet dissuasif.

Dans un premier élan, suivant la loi de finances complémentaire de 20157, les articles 140 et 149 de la loi du 21 avril 1990, concernant respectivement les infractions à l'âge légal du travail et au salaire minimum garanti fixé légalement ou conventionnellement, ont été modifiés, de manière à ce qu'ils soient en harmonie avec les dispositions du Code pénal. Ensuite, par le biais de la nouvelle loi de finances de 2018, le législateur a complété la révision des autres articles de cette même loi ainsi que de celle du 26 janvier 1988 relative à l'hygiène, la sécurité et la médecine du travail. Pour cette loi ${ }^{8}$, l'aménagement a porté sur les articles 37, 38 et 39 puisqu'ils comportent tous des peines d'amendes pour des manquements aux règles de sécurité et d'hygiène sur les lieux du travail et de médecine du travail. Néanmoins, la modification de l'article 38, n'a pas consisté à réviser les montants des amendes, elle a aussi remanié partiellement son contenu. En effet, tandis que dans son ancienne rédaction le texte prévoyait que : « tout contrevenant aux dispositions des articles $3,5,6,7,11,13,14,17,23,24,25,26$ et 28 ci-dessus est passible d'une amende.... », le nouveau texte ne reprend pas l'article 3 qui fait obligation à l'employeur d'assurer l'hygiène et la sécurité aux travailleurs, dans le cadre d'une pénalisation par renvoi, le privant ainsi de toute protection pénale.

Cette omission du législateur algérien (à dessein ou non) risque d'entraver sérieusement la mission des inspecteurs du travail. Elle les empêche, assurément, de constater les manquements aux règles d'hygiène et de salubrité au sein des entreprises, tel que prévu dans les articles $3^{9}$ et $4^{10}$ de la loi $n^{\circ} 88-07$. Par conséquent, cette nouvelle mouture réduit considérablement le pouvoir pénal des inspecteurs du travail, en le limitant aux infractions touchant seulement à la sécurité des travailleurs, conformément à l'article $5^{11}$ de la même loi (étant donné qu'il a été maintenu dans l'article 38 révisé); en revanche, elle leur ôte le droit de constater et de relever les infractions pénales ayant trait aux conditions d'hygiène et de salubrité, suite au non renvoi de l'article 38 à l'article 3.

C'est pourquoi, il aurait mieux fallu, maintenir parmi les textes mentionnés dans l'article 38 de la loi 88-07, l'article 3 et y ajouter aussi l'article 4 qui définit les conditions d'hygiène et de salubrité sur les lieux de travail, ou alors, pour plus de clarté et de précision, il eût mieux valu éviter l'incrimination par renvoi, en énonçant directement dans le texte de loi le fait incriminé et la sanction pénale y relative.

7 Voir loi n ${ }^{\circ}$ 15-01 du 23/07/2015, portant loi de finances complémentaire de 2015, JORA n 40/2015.

8 Voir loi n 88-07 du 26/01/1988, relative à l'hygiène, à la sécurité et à la médecine du travail.

9 Voir art. 3 de la loi n 88-07 : « L'organisme employeur est tenu d'assurer l'hygiène et la sécurité aux travailleurs ».

10 Voir art. 4 de la loi n 88-07 : « Les locaux affectés au travail, les emplacements de travail et leurs environnements, leurs dépendances et leurs annexes, y compris les installations de toute nature mises à la disposition des travailleurs doivent être tenus dans un état constant de propreté et présenter les conditions d'hygiène et de salubrité nécessaires à la santé des travailleurs ... ».

11 Voir art. 5 de la loi n 88-07: : Les établissements, les locaux affectés aux travailleurs dépendances et leurs annexes visés à l'article 4 ci-dessus doivent être conçus, aménagés et entretenus de manière à garantir la sécurité des travailleurs ... ». 


\section{AlgéRIE}

Par ailleurs, et comme signalé précédemment, la nouvelle loi de finances modifie et complète, dans sa partie pénale, la loi 90-11 relative aux relations de travail, en concordance avec les dispositions de l'article 5 du Code pénal. Il faut remarquer, que le législateur al gérien a fixé des amendes et des peines de prison d'une grande sévérité, pour toute infraction aux dispositions de la législation sur les relations du travail individuelles et collectives.

Dans certains cas, l'amende a atteint cinq cent mille (500.000) dinars, sachant que le salaire national minimum garanti est actuellement de dix-huit mille (18.000) dinars, pour tout récidiviste signataire d'une négociation collective comportant une discrimination à l'encontre des travailleurs en matière d'emploi, de rémunération ou de conditions de travail12. Quant à la peine de prison, le contrevenant risque jusqu'à six (6) mois d'emprisonnement en cas de récidive aux infractions à l'obligation de versement à terme échu de la rémunération ${ }^{13}$ ainsi qu'en cas de récidive à l'entrave à la constitution et au fonctionnement du comité de participation (équivalant du comité d'entreprise en France) ${ }^{14}$.

Néanmoins, il est aisé de constater que cet aménagement du droit social algérien nous laisse le sentiment d'un travail inachevé et mal fait. D'abord, du fait de l'utilisation inappropriée de la loi de finances comme support juridique pour modifier et compléter des dispositions du droit du travail et de la sécurité sociale. Ensuite, cet aménagement ne peut être que lacunaire, car il ne contient pas toutes les dispositions du droit du travail et de la sécurité sociale qui ont un grand besoin d'être révisées. II reste entre autres lois, celles qui concernent le droit syndical ${ }^{15}$ et le droit de grève ${ }^{16}$, qui comportent des dispositions pénales totalement désuètes, et qui gagneraient à être amendées.

Par conséquent, on ne peut qu'appeler de nos vœux, la promulgation de l'avant-projet du Code du travail, qui sommeille depuis des années au ministère du travail, et qui a l'avantage de rassembler l'ensemble des législations du travail en Algérie. Ainsi, toutes les dispositions pénales du droit du travail seraient amendées d'un seul coup et avec harmonie. C'est sans nul doute la solution idoine pour mettre fin à toute cette anarchie juridique.

12 Voir art. 142 de la loi n 90-11 du 21/04/1990, relative aux relations de travail, JORA nº 17/1990.

13 Voir art. 150 de la loi $n^{\circ} 90-11$.

14 Voir art. 151 de la loi n ${ }^{\circ}$ 90-11.

15 Voir loi $n^{\circ}$ 90-14 du 02/06/1990, relative aux modalités d'exercice du droit syndical, JORA $n^{\circ} 23 / 1990$

16 Voir loi n 90-02 du 06/02/1990, relative à la prévention et au règlement des conflits collectifs de travail et à l'exercice du droit de grève, JORA nº 06/1990. 